\title{
In Situ Mechanical Testing in Electron Microscopes: Current Progress and Future Opportunities in Small-Scale Experimentation
}

\author{
AMIT PANDEY ${ }^{1,2}$ \\ 1.-Rolls Royce LG Fuel Cell System, North Canton, OH 44720, USA. 2.-e-mail: dramitpandey@ \\ gmail.com
}

The overriding motivation behind conducting small-scale in situ experiments in electron microscopes is to establish a one-to-one correlation between the changes within the microstructure (in real time) of a specimen "in its original place" and its overall deformation response (stress-strain, fracture, etc.). This can enable precise spatial and temporal correlation of slip, fracture, and failure events as a result of plastic flow anywhere within the entire test specimen. ${ }^{1}$ This stands in contrast to macroscopic in situ experiments that characterize a fraction of the entire volume undergoing deformation.

Much of the in situ mechanical testing conducted on small volumes has been directed toward testing specimens in the form of thin fibers and whiskers, microcompression and nanocompression pillars, and thin films. ${ }^{2-5}$ This is sometimes driven by the need to characterize the mechanical properties of a material at intrinsically small volumes for specific applications, e.g., electronics [microelectromechanical systems (MEMS) and nanoelectromechanical systems (NEMS)], ${ }^{6}$ aerospace (coating and superalloys), ${ }^{7}$ energy (solid oxide fuel cell ${ }^{8}$ and filtration ${ }^{9}$ ), etc. Other mechanistic investigations of small scale deformation have benefitted from recent progress in specimen fabrication made possible by the advancement of focused ion beam (FIB) technology ${ }^{10}$ with its versatile milling capabilities at the micron and submicron scale.

So what is next for in situ mechanical testing? The coming years will see the integration of various experimental techniques ${ }^{11}$ accelerating our realtime study of deformation mechanisms. Investigations have now been focused on novel microscale

Amit Pandey is the guest editor for the Advanced Characterization, Testing and Simulation Committee of the TMS Structural Materials Division, and coordinator of the topic In-Situ Mechanical Testing in Electron Microscopes: Part I in this issue. specimen preparation techniques-e.g., batch fabrication-thereby reducing the cost and/or improving statistical sampling of test results. The new plasma FIB instruments also offer dramatic improvements in specimen production throughput while employing an inert ion sputtering source. ${ }^{12,13}$ Perhaps first and foremost, we can expect to observe the convergence of nanoscale and microscale $(5 \mathrm{~nm}$ to $10 \mu \mathrm{m})$ and macroscale $(>1000 \mu \mathrm{m})$ specimen testing in the form of mesoscale specimen (10$1000 \mu \mathrm{m})$ testing. Specimens tested at these length scales can return properties representative of the bulk, while at the same time allowing for observation of the entire specimen volume (or representative bulk volume) in the electron microscope. These tests not only provide bulk property measurement but are amenable to full volume plasticity modeling where calculations of explicit, discrete grain configurations composed of tens or hundreds of grains have become a tractable problem. All these advancements will also promote the use of in situ testing within the electron microscope for industry where results can directly influence design considerations.

This special topic of "In Situ Mechanical Testing in Electron Microscopes" presents a collection of selected articles from well-known researchers all across the world. This topic covers a broad area in the recent advances in in situ testing, techniques, and recent results. In addition, review articles will certainly hold the interest of readers. Details of the articles in this topic are mentioned below. To download any of the papers, follow the URL: http:// link.springer.com/journal/11837/67/8/page/1 to the table of contents page for the August 2015 issue (vol. 67 , no. 8).

1. "Comparison of In Situ Micromechanical Strain Rate Sensitivity Measurement Techniques," by Juri Wehrs, Gaurav Mohanty, Gaylord Guillon- 
neau, Aidan A. Taylor, Xavier Maeder, Damian Frey, Laetitia Philippe, Stefano Mischler, Jeffrey M. Wheeler, and Johann Michler, presents an interesting comparison study on the strain-rate sensitivity of nanocrystalline nickel investigated using three independent, in situ transient experimental techniques: miniature tension, nanoindentation, and micropillar compression.

2. "Evaluation of Incoherent Interface Strength of Solid-State Bonded Ti64/Stainless Steel Under Dynamic Impact Loading," by Devendra Verma, Jogender Singh, Amit H. Varma, and Vikas Tomar, presents unique dynamic indentation experiments on solid diffusion bonded Ti/steel at a smaller scale.

3. "In Situ TEM Microcompression of Single and Bicrystalline Samples: Insights and Limitations," by P. J. Imrich, C. Kirchlechner, D. Kiener, and Gerhard Dehm, demonstrates the benefits and challenges of micromechanical experiments in transmission electron microscopy (TEM), with a special focus on miniaturized samples containing a single grain boundary.

4. "In Situ Microstructural Control and Mechanical Testing Inside the Transmission Electron Microscope at Elevated Temperatures," by Baoming Wang and M. A. Haque, demonstrates the use of a multifunctional microchip to conduct mechanical testing at elevated temperatures. In addition, the chip can actively control microstructures of the specimen material.

5. "A TEM In Situ Straining Technique to Directly Observe Defects and Interfaces during Deformation in Magnesium," by B. M. Morrow, E. K. Cerreta, R. J. McCabe, and C. N. Tomé, shows an in situ straining technique in TEM to study twin-boundary motion in hexagonal closepacked magnesium.

6. In "Combination of Different In Situ Characterization Techniques and Scanning Electron Microscopy Investigations for a Comprehensive
Description of the Tensile Deformation Behavior of a CrMnNi TRIP/TWIP Steel," the authors, Anja Weidner and Horst Biermann, present an overview of the tensile deformation behavior of a low-carbon, high-alloy $\mathrm{CrMnNi}$ steel using different in situ characterization techniques.

7. "In-Situ Scanning Electron Microscopy Observations of Contraction Twinning and Double Twinning in Extruded Mg-1Mn (wt.\%)," by A. Chakkedath and C. J. Boehlert, presents in-situ SEM observations of a magnesium alloy under tension at $323 \mathrm{~K}\left(50^{\circ} \mathrm{C}\right)$.

8. The final article in this topic is by Guilhem Martin, Chad W. Sinclair, Warren J. Poole, and Hamid Azizi-Alizamini, who present their work on "Local Plastic Strain Heterogeneities and Their Impact on the Ductility of Mg." This article illustrates the use of microscale digital image correlation based on scanning electron microscope images to reveal plastic heterogeneity in commercially pure polycrystalline $\mathrm{Mg}$.

\section{REFERENCES}

1. R. Wheeler, D. Bhattacharya, A. Pandey, A. Shyam, A. Shiveley, and D. Sergison, Microsc. Microanal. 20, 1466 (2014).

2. S.S. Brenner, J. Appl. Phys. 27, 1484 (1956).

3. D.S. Gianola and C. Eberl, JOM 61, 24 (2009).

4. M.A. Haque and M.T.A. Saif, Exp. Mech. 42, 123-128 (2002).

5. M.D. Uchic, D.M. Dimiduk, J.N. Florando, and W.D. Nix, Science 305, 986 (2004).

6. N.B. Jaya and M.Z. Alam, Curr. Sci. 105, 1073 (2013).

7. A. Pandey, V.K. Tolpygo, and K.J. Hemker, JOM 65, 542 (2013)

8. A. Pandey, A. Shyam, Z. Liu, and R. Goettler, J. Power Sources 273, 522 (2015).

9. A. Pandey, A. Shyam, T.R. Watkins, E. Lara-Curzio, R.J. Stafford, and K.J. Hemker, J. Am. Ceram. Soc. 97, 899 (2014).

10. C.A. Volkert and A.M. Minor, MRS Bull. 32, 389 (2007).

11. M. Schoßig, A. Zankel, C. Bierögel, P. Pölt, and W. Grellmann, Compos. Sci. Technol. 71, 257 (2011).

12. P.A. Shade, S.L. Kim, R. Wheeler, and M.D. Uchic, Rev. Sci. Instrum. 83, 053903 (2012).

13. R. Wheeler, A. Pandey, A. Shyam, T. Tan, and E. Lara-Curzio, Exp. Mech. (2015). doi:10.1007/s11340-015-0020-6. 